\title{
Bis ins sechste Lebensjahr nachweisbar
}

Fragestellung: Wie lange ist der negative Effekt auf Kognition und neuropsychologische Entwicklung bei Kindern, deren Mütter während der Schwangerschaft antikonvulsive Medikamente (AED) einnehmen mussten, nachweisbar?

Hintergrund: Dass AED, die während der Schwangerschaft eingenommen werden, mit einem erhöhten Missbildungsrisiko beim Ungeborenen einhergehen können, ist bekannt. Die Missbildungsrate und -schwere hängt vom Präparat, aber auch maßgeblich von der Dosis ab. Aus Tiermodellen weiß man, dass es bereits in Dosierungen, die unter dem regulären Missbildungsrisiko liegen, zu kognitiven Störungen und Verhaltensauffälligkeiten bei den Jungen kommen kann. Außerdem zeigen diese Tiere regelhaft ein signifikant reduziertes Hirngewicht.

Patienten und Methodik: In diese prospektive und multizentrische Beobachtungsstudie wurden schwangere EpilepsiePatientinnen eingeschlossen, die eines der gängigen AED

Meador KJ, Baker GA, Browning $\mathrm{N}$, et al; for the NEAD Study Group. Fetal antiepileptic drug exposure and cognitive outcome at age 6 years (NEAD study): a prospective observational study. Lancet Neurol 2013; 12: $244-52$ in Monotherapie einnahmen: Carbamazepin (CBZ), Lamotrigin (LTG), Phenytoin (PHT) oder Valproat (VPA). Mütter mit einem IQ unter 70 wurden prinzipiell ausgeschlossen. Weitere Ausschlusskriterien waren unter anderem Medikamentenmissbrauch, antikonvulsive Polytherapien oder infektiologische Zusatzdiagnosen (u. a. HIV, Lues). Die Compliance der Mütter wurde streng überwacht, auch anhand von Medikamentenspiegelkontrollen. Von zunächst 305 Müttern und 311 Kindern beendeten 224 Kinder das 6-JahresFollow-up, in dem in definierten Abständen entsprechende neurokognitive, Sprach- und Entwicklungstests erfolgten.

Ergebnisse: Alle Kinder, deren Mütter während der Schwangerschaft VPA eingenommen hatten, zeigten signifikante geringere IQ-Werte als Kinder, die einem der drei anderen Antikonvulsiva ausgesetzt waren. So lag der durchschnittliche IQ bei Kindern, deren Mütter LTG erhalten hatten, bei 109, bei Kindern in der PHT-Gruppe bei 106, in der CBZ-Gruppe bei 106 und in der VPA-Gruppe bei 94. Ähnlich sahen die Ergebnisse für das Verbalgedächtnis und die Memory-Tests aus. Interessanterweise waren die LTG-Kinder in den non-verbalen Tests und den exekutiven Funktionen gleich schlecht wie die VPA-Kinder im Gegensatz zur Gruppe unter CBZ oder PHT. Kinder, deren Mütter Folsäure perikonzeptionell genommen hatten, hatten generell im Schnitt einen höheren IQ.

Schlussfolgerungen: Kinder, die intrauterin Valproinsäure ausgesetzt waren, zeigen bis in das sechste Lebensjahr nachweislich einen signifikant reduzierten IQ im Vergleich zu Gleichaltrigen, außerdem Störungen des Verbalgedächtnisses und eine Veränderung der Lateralisation der Hemisphären.

\section{- Kommentar von Vivien Homberg, Bad Berka}

\section{Betroffene Kinder frühzeitig intellektuell fördern}

Im Vergleich zu einer früheren Publikation der Gruppe, die 2009 die Drei-Jahresdaten veröffentlicht hatte, liegen uns nun die Daten aus sechs Jahren Beobachtungszeit nach intrauteriner antikonvulsiver Medikamentenexposition vor. Erschreckend: Die Kinder haben zwar insgesamt etwas aufgeholt über die Jahre, aber eben nicht vollständig, vor allem die VPA-exponierten Kinder zeigen fast unverändert schlechte IQ-Ergebnisse und Verbalgedächtnisstörungen. VPA ist jedoch bei weitem nicht alleinig das Problem, alle haben, wenn auch sehr milde kognitive Beeinträchtigungen über die Jahre behalten und es ist wenig wahrscheinlich, dass sich das noch „verwächst". Besonders dramatisch sind, da nicht erwartet, die schlechten Ergebnisse nach LTG-Exposition in den non-verbalen Tests und den exekutiven Funktionen. Wenn man bedenkt, dass die Studienkinder in spezielle edukative Programme eingeschlossen wurden, Lerneffekte oder Routine bei so häufigen Testuntersuchungen nicht auszuschließen sind, sind die Endergebnisse bei nicht geförderten Kindern noch weit darunter zu vermuten. Ein kleiner Trost ist die Tatsache, dass Folsäure auch hierfür ein guter Begleiter ist. Nicht nur weil sie, wie mehrfach belegt, vor Missbildungen schützt, sondern auch vor kognitiven Beeinträchtigungen.
Auch wenn diese Studie einige Einschränkungen aufweist, gilt weiterhin: Vorsicht mit dem was man verordnet und in welcher Dosis. Andererseits kann nur immer wieder vor hektischen Umsetzversuchen gewarnt werden, was die Schwangere bezüglich ihrer Epilepsie unnötig gefährdet (Stürze), die Fahrerlaubnis durch Anfallsrezidive kostet und mehr. Was wir sicher mitnehmen sollten ist, dass scheinbar gesund Geborene eine besondere intellektuelle Förderung erhalten sollten, damit das kognitive Defizit, was nicht so offensichtlich vor uns liegt, frühzeitig beübt wird und nicht erst in der Schule zu Problemen führt.

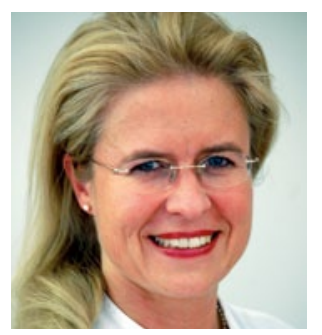

Dr. med. Vivien Homberg, Bad Berka

Chefärztin der Klinik für Neurologie Zentralklinikum Bad Berka E-Mail: Vivian.Homberg@zentralklinik.de 